\title{
ROTOR-STATOR INTERACTION INDUCED PRESSURE FLUCTUATIONS: CFD AND HYDROACOUSTIC SIMULATIONS IN THE STATIONARY COMPONENTS OF A MULTISTAGE CENTRIFUGAL PUMP
}

\author{
Stefan Berten \\ Laboratory for Hydraulic Machines - EPFL \\ Lausanne, Switzerland \\ Email: stefan.berten@epfl.ch \\ Mohamed Farhat \\ Laboratory for Hydraulic Machines - EPFL \\ Lausanne, Switzerland \\ Email: mohamed.farhat@epfl.ch
}

\author{
Philippe Dupont \\ Sulzer Pumps Ltd. \\ Winterthur, Switzerland \\ Email: philippe.dupont@sulzer.com \\ Francois Avellan \\ Laboratory for Hydraulic Machines - EPFL \\ Lausanne, Switzerland \\ Email: francois.avellan@epfl.ch
}

\section{ABSTRACT}

In a centrifugal pump the interaction between the rotating impeller pressure field and the stationary diffuser pressure field yields pressure fluctuations as the result of a modulation process. These fluctuations may induce hydroacoustic pressure fluctuations in the exit chamber of the pump and could cause unacceptable vibrations. This paper presents a methodology for the prediction of hydroacoustic pressure fluctuations resulting from rotor-stator interaction in a multistage centrifugal pump. The method consists in the one-way coupling of incompressible CFD and hydroacoustic simulations. In a first step the rotorstator pressure fluctuations are calculated using a commercial 3D-RANS CFD-code (CFX 10) for different flow rates. The acoustic simulations are performed in two consecutive steps. Initially a free oscillation analysis using white noise pressure fluctuations is performed, which provides hydroacoustic eigen frequencies and mode shapes of the outlet casing. In a second step the spatially distributed pressure fluctuations from the CFD simulation are used to perform a forced oscillation analysis. This approach allows the prediction of possible standing waves in the hydraulic collection elements in the last stage of multistage pumps.
NOMENCLATURE

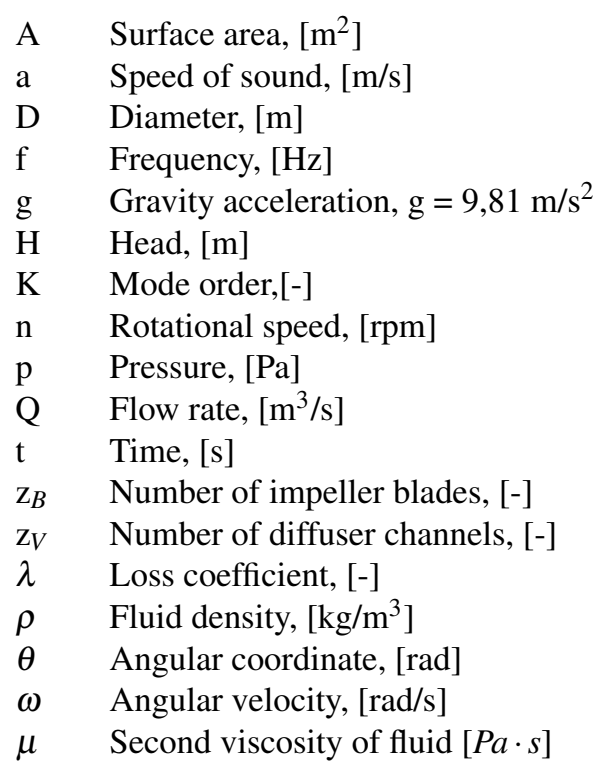




\section{INTRODUCTION}

Harmful vibratory levels of high specific power pumps can be caused by pressure fluctuations resulting from rotor-stator interactions (RSI) influenced by the vibratory characteristics of the structure and the acoustic properties of the flow passages. These vibrations could compromise the mechanical integrity of rotating and stationary components of the machine [1].

The first description of the rotor-stator interaction pressure fluctuations has been made by Den Hartog in 1930 [2]. More detailed attempts to describe these pressure fluctuations date back to the 1960ies. Domm and Dernedde [3] derived simple rules for the selection of impeller and diffuser vane numbers from the assumption of periodically fluctuating pressures without taking into account their amplitudes. Bolleter [4] summarized the sources of blade passing tones in centrifugal pumps and gave recommendations for vane number combinations. According to him, only small order diametral modes are problematic and should be therefor avoided. Tanaka [5] presented measurement results of vibrations in pump turbines which were excited by RSI induced pressure fluctuations and explained the interaction between RSI modes and mechanical vibrations of rotating and stationary components. Franke et al. [6] used the operational deflection shape analysis, usually applied in modal analysis to measure the pressure modes arising from rotor-stator interaction.

The phenomenon of standing waves in spiral casings of storage pumps has been first modeled by Chen [7], who derived a criterion to avoid these standing waves. Dörfler [8] discussed the model of Chen and proposed a modeling of the hydraulic passages with pipe elements and calculating the transfer matrices for these elements.

Haban et al. [9] used the transfer matrix method to calculate the pressure fluctuations in a pump turbine. They included as well the influence of the second viscosity of water [10] and showed by comparing with experimental results that it may have a significant influence. Nicolet et al. [11] simulated the rotorstator interaction phenomenon in a Francis pump turbine in the time domain. They used a network of pipes and valves with an appropriate closing sequence. With this simplified excitation model, a parametric study has been performed.

The present paper presents the results of a simulation of the hydroacoustic response of a centrifugal pump outlet chamber to rotor-stator interaction generated pressure fluctuations using an improved excitation model. First, the phenomenon of rotor-stator interaction is explained and the expected RSI modes for the machine are calculated. Then the results of an unsteady CFD simulation of the stage are presented. The pressure fluctuation amplitudes obtained from this simulation are then applied in a 1D model of the stationary components and the response of these components is analyzed.

\section{ROTOR-STATOR INTERACTIONS}

The rotating pressure/velocity field leaving the impeller of a centrifugal pump is interacting with the stationary components downstream. This interaction causes periodic pressure fluctuations at specific frequencies which are multiples of the blade passing frequency. The pressure can be described as the sum of the mean pressure $\bar{p}$ and the fluctuating part p'. The interaction between the rotating field at the angular coordinates $\theta_{B}$ and the stationary field at the angular coordinates $\theta_{V}$ can be described as a modulation as follows. The pressure fields in their respective reference frames can be decomposed into Fourier series

$$
\begin{aligned}
p_{B}^{\prime} & =\sum_{m=1}^{\infty} p_{m} \cdot \cos \left(m \cdot z_{B} \cdot \theta_{B}\right) \\
p_{V}^{\prime} & =\sum_{n=1}^{\infty} p_{n} \cdot \cos \left(n \cdot z_{V} \cdot \theta_{V}\right)
\end{aligned}
$$

and its modulation is calculated according to Bolleter [4] using

$$
p^{\prime}(\theta, t)=\sum_{n, m=1}^{\infty} p_{n} \cdot \cos \left(n \cdot z_{V} \cdot \theta_{V}\right) \cdot p_{m} \cdot \cos \left(m \cdot z_{B} \cdot \theta_{B}\right)
$$

Rotating and stationary domain are related to each other through

$$
\theta_{V}=\theta_{B}-\omega \cdot t
$$

yielding the time dependent pressure field in the stationary frame of reference

$$
\begin{aligned}
p^{\prime}\left(\theta_{V}, t\right)= & p_{n} \cdot \cos \left(m \cdot z_{B} \cdot \omega \cdot t-\left(m \cdot z_{B}-n \cdot z_{V}\right) \theta_{V}\right) \\
& +p_{m} \cdot \cos \left(m \cdot z_{B} \cdot \omega \cdot t-\left(m \cdot z_{B}+n \cdot z_{V}\right) \theta_{V}\right)
\end{aligned}
$$

By this equation 2 diametral pressure modes having the following number of nodes [6]:

$$
K_{1}=m \cdot z_{B}-n \cdot z_{V} \quad \text { and } \quad K_{2}=m \cdot z_{B}+n \cdot z_{V}
$$

can be found. These modes are rotating with the angular velocities $\omega_{1}$ and $\omega_{2}$ in the stationary reference frame ( [6]):

$$
\omega_{1}=\frac{m \cdot z_{B}}{K_{1}} \cdot \omega \quad \text { and } \quad \omega_{2}=\frac{m \cdot z_{B}}{K_{2}} \cdot \omega
$$

The lower the mode order $\mathrm{K}$ is, the higher amplitudes are expected [4]. The configuration analyzed here is a typical last stage 
Table 1. EXPECTED ROTOR-STATOR INTERACTION PATTERNS FOR THE VANE NUMBER COMBINATION $z_{B}=7$ and $z_{V}=12$

\begin{tabular}{ccccc}
\hline & & \multicolumn{3}{c}{ Stationary frame } \\
$\mathrm{m}$ & $\mathrm{n}$ & $\mathrm{K}_{1}$ & $\omega_{1} / \omega_{n}$ & $f / f_{n}$ \\
\hline 1 & 2 & 2 & 7 & 14 \\
2 & 3 & -3 & -7 & 21 \\
2 & 4 & 4 & 7 & 28 \\
3 & 5 & -1 & -35 & 35 \\
3 & 6 & 6 & 7 & 42 \\
\hline
\end{tabular}

arrangement of a high energy multistage centrifugal pump as it can be found in various applications. This type of machines often operates with variable speed drives. This speed variation increases the risk to pass through acoustical resonances which could lead to high vibratory levels.

The analyzed stage consists of an impeller with $\mathrm{z}_{B}=7$ vanes, a diffuser with $\mathrm{z}_{V}=12$ channels leading into an annular collection chamber with a constant cross section connected to the discharge nozzle (see Fig.1). Equation 5 allows the calculation of the rotorstator interaction patterns as presented in Tab.1.

\section{CFD SIMULATION}

A fully unsteady CFD simulation of the last stage consisting of the impeller, diffuser, annular chamber and the discharge pipe has been performed in order to calculate the pressure fluctuations at the diffuser inlets which will be used as input for the acoustic simulations.

\section{Mesh, Boundary Conditions and Convergence Criteria}

The CFD simulation has been performed using CFX10 with block structured meshes. The boundary conditions and calculation domains can be found in Fig.1. 4 different mesh variations have been generated in order to study the influence of the mesh density on mean values of pressure and torque as well as on the local pressure fluctuation amplitudes. Based on the results of this analysis, finally a mesh with 1'446'795 elements has been used to perform the unsteady simulation. At the inlet the mass flow rate and the flow angle, at the outlet a constant average pressure have been specified. Between rotating and stationary components a transient rotor-stator interface has been applied. After obtaining an initial solution using a frozen rotor approach, 420 angular steps per revolution have been calculated. The calculation has been performed at the best efficiency point. The con-

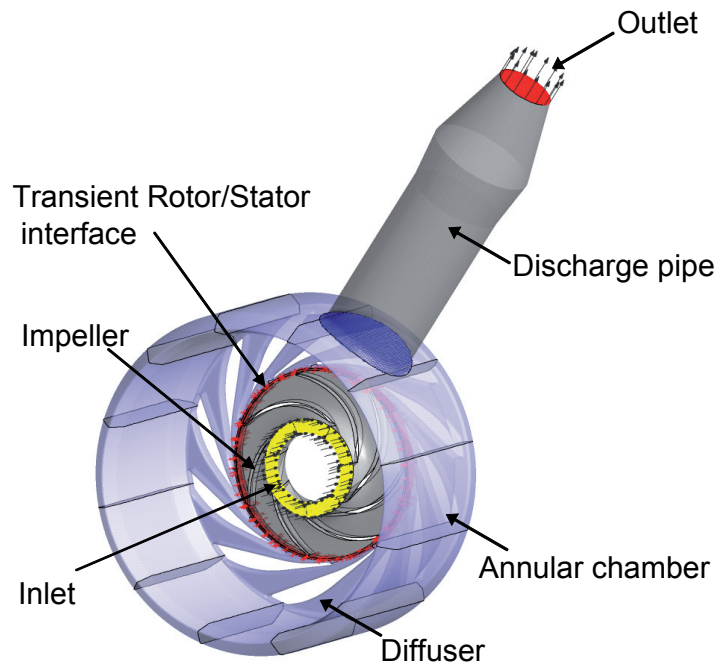

Figure 1. CFD MODEL AND BOUNDARY CONDITIONS

vergence of the simulation has been traced analyzing the global values (pressure coefficient and blade torque) as well as the local pressure fluctuations in positions of interest. Doing this, a convergence criterion has been established similar to the one proposed by Clark and Grover [12]. Here, not only mean values but also amplitude and phase spectra and the cross-correlation between two consecutive periods are taken into account to judge the convergence of the simulation.

\section{Pressure Fluctuations in Stationary Components}

Instantaneous pressure values have been recorded during the simulation in several positions in the rotating and stationary domains. These values are used to check the convergence of the CFD simulation, to compare the resulting pressure fluctuations with the expected rotor-stator interaction pattern and finally the pressure fluctuations in the diffuser inlets are used to derive the excitation signals for the hydroacoustic simulation. In order to simplify the data transfer from the CFD simulation to the acoustic simulation the pressure fluctuations in the diffuser have been decomposed into Fourier series. The time domain signal can be recomposed with

$$
f(t)=\frac{a_{0}}{2}+\sum_{n=1}^{\infty}\left(a_{n} \cdot \cos (n \omega t)+b_{n} \cdot \sin (n \omega t)\right)
$$

The decomposition has been performed for discrete multiples of the rotational angular velocity $\omega_{n}$ at $\omega / \omega_{n}=$ $\left[\begin{array}{lllllll}1 & 7 & 14 & 21 & 28 & 35 & 42\end{array}\right]$. These frequency components have been selected, because they are the resulting frequencies 

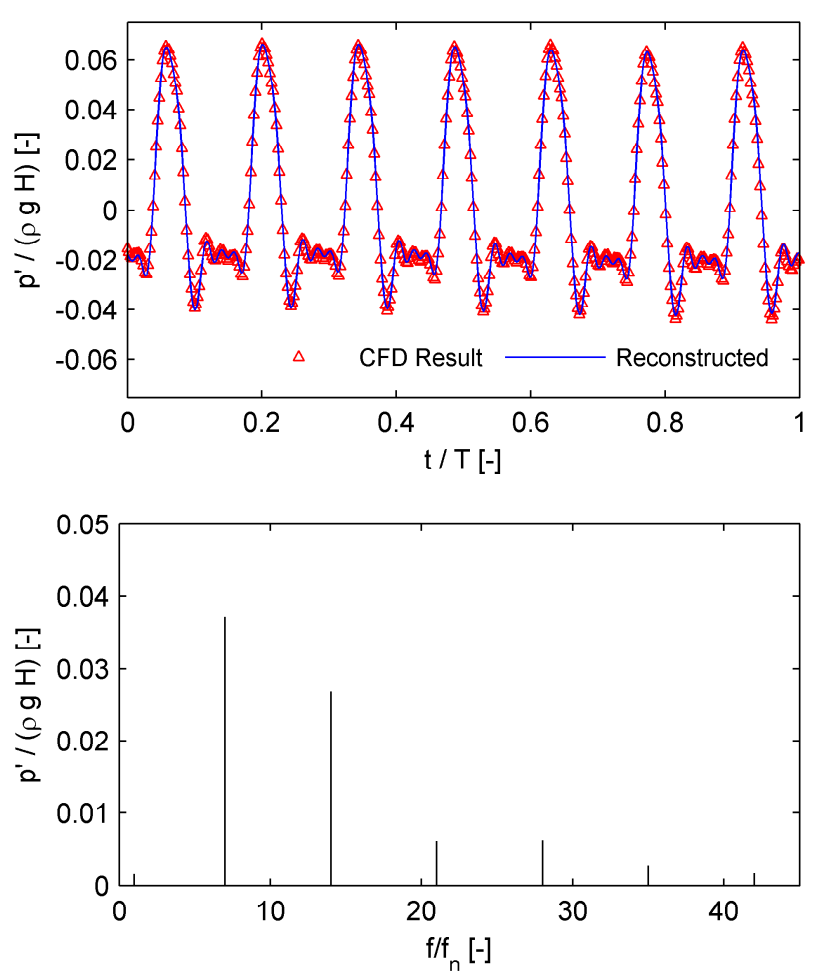

Figure 2. TIME DEPENDEND PRESSURE FLUCTUATIONS AND CALCULATED AMPLITUDE SPECTRUM IN THE DIFFUSER INLET

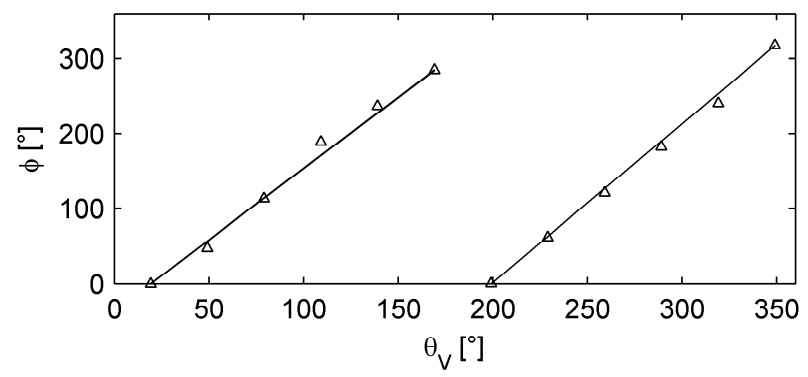

Figure 3. PHASE OF PRESSURE FLUCTUATIONS $\phi$ AS FUNCTION OF THE ANGULAR POSITION $\theta$ FOR THE MODE $\mathrm{K}=+2, f / f_{n}=14$

for small $\mathrm{K}_{1}$ according to Tab.1. Figure 2 shows the comparison between the reconstructed and the original pressure fluctuations, normalized to the mean pressure difference over the stage and the dimensionless pressure fluctuation amplitudes p'. As it can be seen, these 6 components are sufficient to reproduce the original signal, the maximum error was $0.26 \%$. Tracing the phase angles in the different diffuser inlets allows the evaluation of the modes in the stationary domain. Figure 3 shows the phase eval- uation for the mode with the order $\mathrm{K}_{1}=2\left(\mathrm{f} / \mathrm{f}_{n}=14\right)$. The slope of the lines connecting the points is positive, larger 1 indicating that this mode is positive and rotating in the same direction as the impeller.

\section{HYDROACOUSTIC SIMULATIONS}

The acoustic behavior of the stationary components is simulated using the EPFL software SIMSEN. This package, originally developed for the analysis of electrical networks, has been extended to hydraulic systems and used for the simulation of the transient behavior of piping systems and hydraulic machinery ( [13] [14]) and for free and forced oscillation analysis of piping systems [15]. In the present study the pressure fluctuations obtained from the CFD simulation are applied directly to pressure sources placed in the diffuser inlets. This more realistic excitation aims the prediction of the resulting pressure amplitudes in the outlet chamber at different rotational speeds.

\section{Simulation Model}

For the 1D - hydroacoustic analysis the flow field is described by the system of partial differential equations as follows [16].

$$
\left\{\begin{array}{l}
\frac{\partial H}{\partial x}+\frac{1}{g A} \frac{\partial Q}{\partial t}+\frac{\lambda Q|Q|}{2 g D A^{2}}=0 \\
\frac{\partial H}{\partial t}+\frac{a^{2}}{g A} \frac{\partial Q}{\partial x}=0
\end{array}\right.
$$

A pipe as in Fig.4 is represented by a circuit as in Fig.5. The

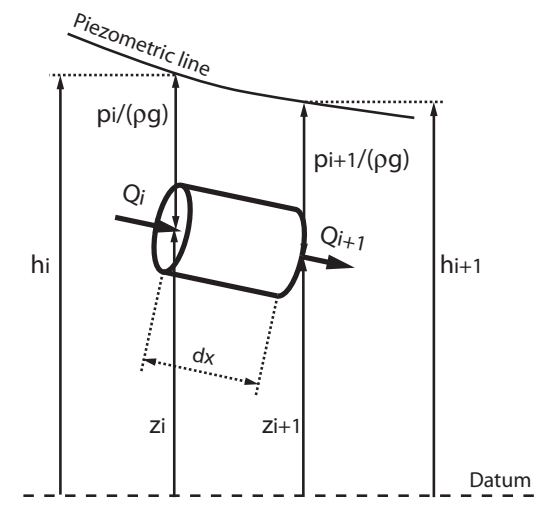

Figure 4. Electrical representation of a pipe

equivalent scheme of a pipe consists of a resistance $\mathrm{R}_{h}$, a viscoelastic resistance $\mathrm{R}_{V} e$, an inductance $\mathrm{L}$ and a capacitance $\mathrm{C}$. 


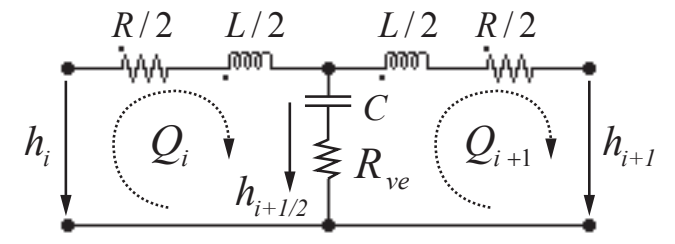

Figure 5. Electrical representation of a pipe

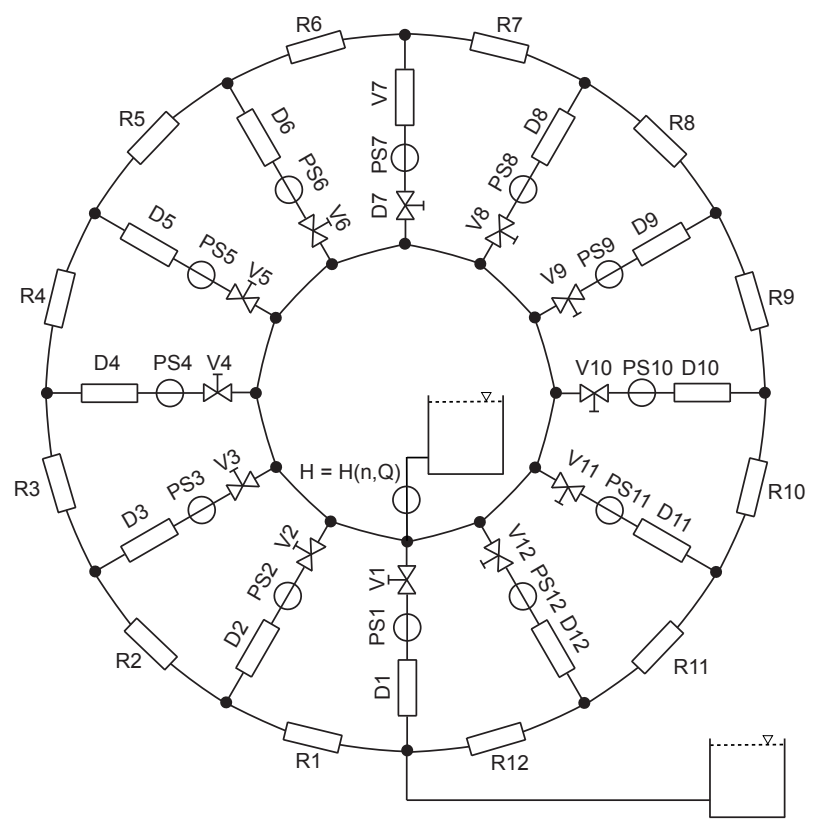

Figure 6. HYDROACOUSTIC MODEL OF THE PUMP STAGE

These parameters account respectively for both losses, inertia and storage effects and are given by:

$$
\begin{aligned}
R_{h}=\frac{\lambda \cdot|Q| \cdot d x}{2 \cdot g \cdot D \cdot A^{2}} & R_{V e}=\frac{\mu}{\rho \cdot g \cdot A \cdot d x} \\
L=\frac{d x}{g A} & C=\frac{g \cdot A \cdot d x}{a^{2}}
\end{aligned}
$$

In the used simulation package, the implicit finite difference method is used. More details can be found in [17]. The hydroacoustic model (Fig.6) consists of 12 pressure sources for the application of the pressure pulsations, $12 \mathrm{semi}$ open valves, which count for the upstream conditions, 12 pipes for the diffuser channels and 12 pipes for the annulus ring. The annular ring is in one position directly connected to a reservoir, this creates a pressure node in this position.

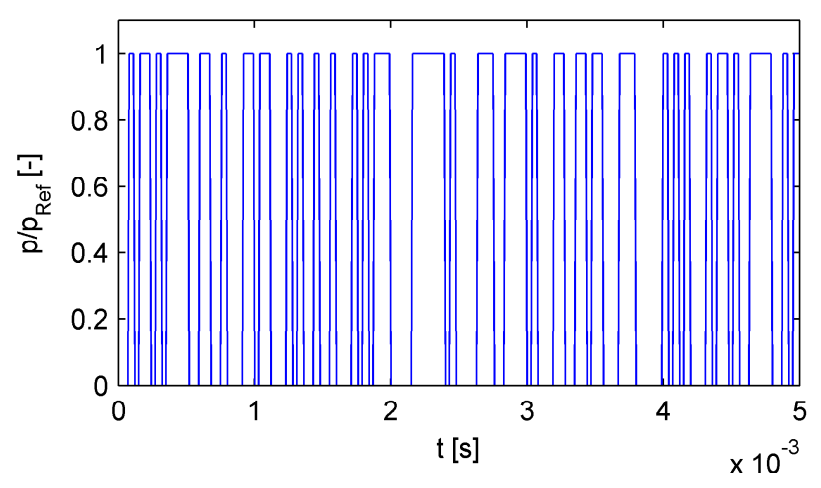

Figure 7. PRBS INPUT SIGNAL IN TIME DOMAIN

\section{Discretization, Boundary Conditions, Fluid Properties}

The spatial discretization of the calculation domain is depending on the frequencies of interest as well as of the hydroacoustic parameters. 9 nodes per diffuser channel and 10 nodes per annular ring section have been used to model a wavelength at 6th order of the impeller vane passing frequency, corresponding to an error on the eigen frequency determination below $1 \%$ [17] at frequencies below the frequency of interest. The RLC parameters of the calculation scheme have been calculated using Eq.9. The viscoelastic resistance has been calculated using values for the second viscosity of water measured by Foldyna et al. [18]. The wave speed in closed channels depends on the mechanical properties of the walls and can be calculated with the following relation:

$$
a^{2}=\frac{1}{\rho\left(\frac{1}{K_{e}}+\frac{\Delta A}{A \cdot \Delta p}\right)}
$$

The term $\Delta A /(A \cdot \Delta p)$ for the different machine components has been evaluated using a conventional FEM package (ANSYS), $\mathrm{K}_{e}$ is the bulk modulus of elasticity of the fluid.

\section{Free Oscillation Analysis}

The acoustic eigen frequencies are determined with a free oscillation analysis in which the acoustic response of the model to an excitation with noise is analyzed. The excitation is applied at 12 diffuser inlets. The noise has been generated with a binary sequence (PRBS) as explained in [19]. The amplitude spectrum of the PRBS shows an even distribution over the frequency range of interest. The resulting amplitudes have been normalized with the mean aplitude of one excitation source, the frequencies are normalized to the first natural acoustic frequency of an open pipe $\mathrm{f}_{0}$ having a length $\mathrm{l}_{e q}$ equal the circumference of the ring chamber 


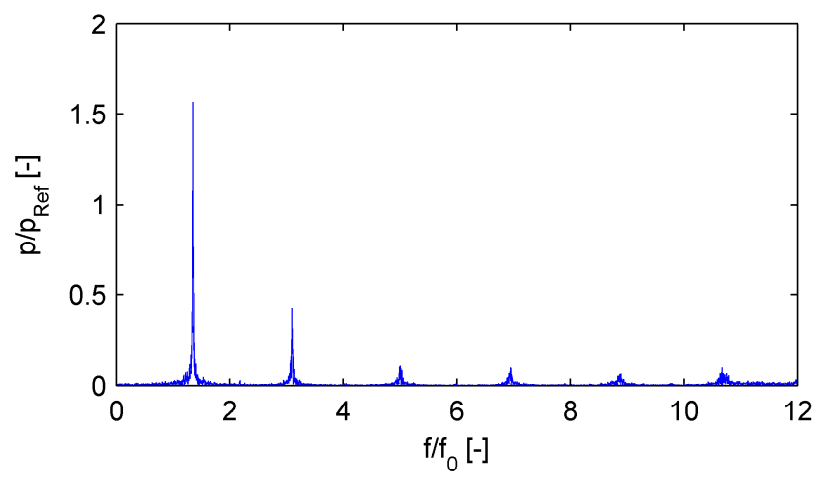

Figure 8. ACOUSTIC RESPONSE IN ONE RING CHAMBER POSITION

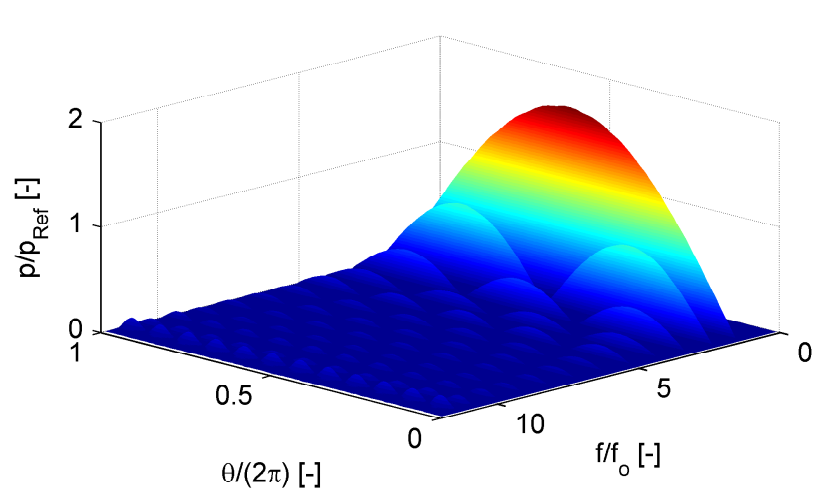

Figure 9. WATERFALL PLOT OF RING CHAMBER PRESSURE PULSATION RESPONSE

mean line calculated with Eq.11.

$$
f_{0}=\frac{a}{2 \cdot l_{e q}}
$$

Figure 8 shows the response in the casing opposite the outlet pipe to the PRBS excitation. Only the odd harmonics of the eigen frequencies are visible here since there is a pressure node at this position. Figure 9 shows the first 12 acoustic eigen modes in the annular chamber. The acoustic eigen modes and frequencies show at low frequencies deviations from the eigen frequencies of a single pipe. This implies that simplified analysis based on developed pipe lengths are in this case not recommendable.

\section{Forced Oscillation Analysis}

The pressure fluctuations obtained using CFD in the diffuser inlets were decomposed into Fourier series. The calculated
Table 2. FREQUENCY AND MODE RELATIONS FOR DIFFERENT SPEEDS

\begin{tabular}{|c|c|c|c|c|c|c|}
\hline \multicolumn{7}{|c|}{ Acoustic } \\
\hline$K_{a}$ & 1 & 2 & 3 & 4 & 5 & 6 \\
\hline$f / f_{0}$ & 1.348 & 2.176 & 3.099 & 4.049 & 5.017 & 5.966 \\
\hline \multicolumn{7}{|c|}{ Rotor-stator interaction } \\
\hline$K_{1}$ & -1 & 2 & -3 & 4 & -5 & 6 \\
\hline \multicolumn{7}{|c|}{ Excitation frequencies at different rotational speeds } \\
\hline \multicolumn{7}{|c|}{$\omega / \omega_{\text {nom }}=\mathbf{1}$} \\
\hline$f / f_{0}$ & 8.724 & 3.49 & 5.234 & 6.979 & 1.745 & 10.469 \\
\hline \multicolumn{7}{|c|}{$\omega / \omega_{\text {nom }}=\mathbf{0 . 8 9}$} \\
\hline$f / f_{0}$ & 7.747 & 3.099 & 4.648 & 6.198 & 1.549 & 9.296 \\
\hline \multicolumn{7}{|c|}{$\omega / \omega_{\text {nom }}=\mathbf{0 . 6 2}$} \\
\hline$f / f_{0}$ & 5.439 & 2.176 & 3.263 & 4.351 & 1.088 & 6.527 \\
\hline
\end{tabular}

coefficients for frequencies up to the 6th harmonic of the impeller blade passing frequency were then used to reconstruct the time dependent pressure fluctuations to be applied on the pressure sources in the diffuser inlets. Figure 2 shows the comparison between the CFD result and the reconstructed signal. With this excitation, 3 cases at different rotational speeds have been calculated. The respective amplitudes and flow rates have been adapted using the similarity laws $\left(Q \sim n, H \sim n^{2}\right)$. Table 2 shows the relation of frequencies and node numbers for the different simulation cases, Fig.10 the simulation results. The amplitudes have been normalized using the amplitude at the impeller blade passing frequency. In the first case a simulation at nominal rotational speed has been performed. For all analyzed frequency components exists a separation between the rotor-stator interaction pressure fluctuations and the acoustic eigen frequencies of the casing. The response amplitudes are very low. In a second case the rotational speed was selected in the way, that a rotorstator interaction mode matches an acoustic eigen frequency. The mode order of the rotor-stator interaction is lower than the acoustic mode order. The casing responds very strongly in the acoustic mode which was excited. In a last case the rotational speed was further reduced that a rotor-stator interaction mode hit an acoustic mode of the same order. Also here the casing responds strongly to the excitation but the amplification is smaller. Figure 10 shows waterfall diagrams of the acoustic response to the excitation. From this it can be concluded, that matching one of the 

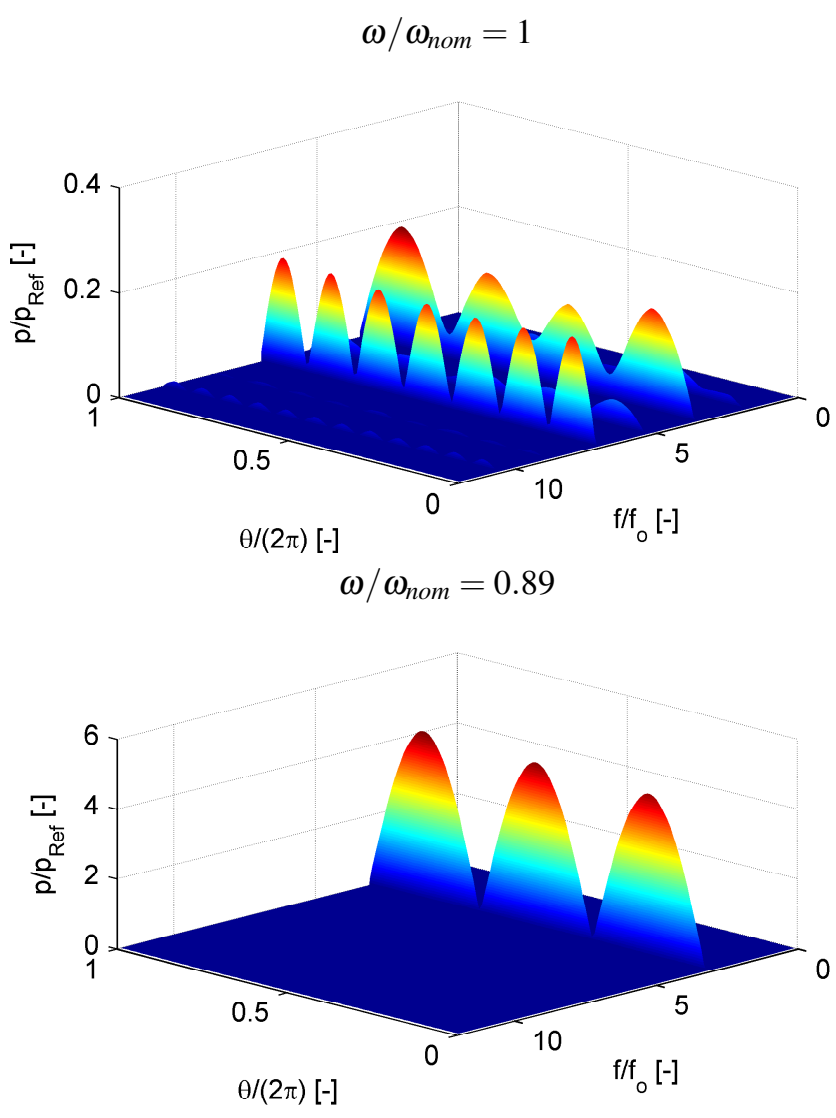

$\omega / \omega_{\text {nom }}=0.62$

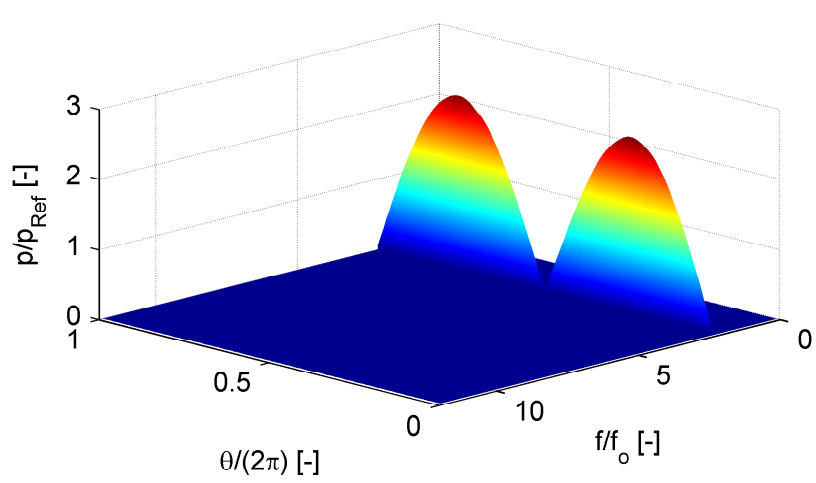

Figure 10. ACOUSTIC RESPONSE IN THE RING CHAMBER AT DIFFERENT ROTATIONAL SPEEDS

acoustic eigen frequencies of the collecting chamber is sufficient to generate a strong acoustic resonance. The identity of the mode order is not necessary.

\section{SUMMARY AND CONCLUSIONS}

The results of the CFD as well acoustic simulations of rotorstator interaction induced pressure fluctuations in the last stage of a high energy multistage centrifugal pump have been presented. The CFD was capable to simulate the rotor-stator interaction induced pressure fluctuations and rotating modes as expected. With the free oscillation analysis it was possible to estimate the acoustic eigen frequencies and modes of standing waves in the outlet chamber. The forced oscillation analysis using the pressure fluctuation resulting from rotor-stator interactions showed that in the case an acoustic eigen frequency is excited, a strong amplification in the outlet chamber occurs. The order of the rotor-stator interaction mode has no influence on the amplification. These results allow to derive recommendations for the operation speeds of such kind of machines.

\section{ACKNOWLEDGMENT}

This study is supported by Sulzer Pumps. The authors wish to thank the Laboratory for Hydraulic Machines, especially Dr. Christophe Nicolet for providing support in the simulation and analysis work and the Electrical Machines Laboratory for providing the simulation package and the support.

\section{REFERENCES}

[1] Eckert, L., 2001. "Radial wheel damages as a result of aeroelastic self excited wheel vibrations". VDI Berichte(1606), pp. 259-278.

[2] Den Hartog, J., 1930. "Mechanical vibrations in penstocks of hydraulic machinery". Trans. Amer. Society of Mechanical Engineers, Hydraulics, pp. 101-110.

[3] Domm, U., and Dernedde, R., 1964. "Über eine Auswahlregel für die Lauf- und Leitschaufelzahl von Kreiselpumpen”. KSB Technische Berichte, 9, pp. 21-23.

[4] U.Bolleter, 1988. "Blade passage tones of centrifugal pumps". Vibrations, 4(3), September, pp. 8-13.

[5] Tanaka, H., 1990. "Vibration behaviour and dynamic stress of runners of very high head reversible pump-turbines". In IAHR Symposium 1990 Belgrade, IAHR.

[6] Franke, G., Powell, C., Seidel, U., Koutnik, J., and Fisher, R., 2003. "On pressure mode shapes arising from rotor stator interactions". In IAHR WG1-2003 Meeting.

[7] Chen, Y., 1961. "Water-pressure oscillations in the volute casings of storage pumps". Sulzer Technical Review (Research Number), pp. 21-34.

[8] Doerfler, P., 1984. "On the role of phase resonance in vibrations caused by blade passage in radial hydraulic turbomachines.". In 12th IAHR Symp. on Hydraulic Machinery in the Energy Related Industries, IAHR, ed., IAHR, Nat. Engng. Lab, pp. 228-241. Session 3, Paper 3.3. 
[9] Haban, V., Koutnik, J., and Pochyly, F., 2002. "1-D mathematical model of high-frequency pressure oscillations induced by RSI including an influence of fluid second viscosity". In Proceedings of the XXIst IAHR Symposium on Hydraulic Machinery and Systems.

[10] Liebermann, L. N., 1949. "The second viscosity of liquids". Phys. Rev., 75(9), pp. 1415-1422.

[11] Nicolet, C., Ruchonnet, N., and Avellan, F., 2006. "Onedimensional modeling of rotor stator interaction in francis pump-turbine". In Proceedings of ISROMAC-11, ASME: International Symposium on Transport Phenomena and Dynamics of Rotating Machinery, Honolulu, Hawaii, USA,.

[12] Clark, J., and Grover, E., 2006. "Assessing convergence in predictions of periodic-unsteady flowfields". In Proceedings of the ASME Turbo Expo, Vol. 6 PART B, pp. 18311841.

[13] Nicolet, C., Avellan, F., Allenbach, P., Sapin, A., and Simond, J., 2002. "New tools for the simulation of transient phenomena in francis turbine power plants". In Proceedings of the 21st IAHR Symposium on Hydraulic Machinery and Systems, pp. 519-528.

[14] Nicolet, C., Greiveldinger, B., Herou, J.-J., Allenbach, P., Simond, J., and Avellan, F., 2006. "On the hydroelectric stability of an islanded power network". In Proceedings IEEE Power Engineering Society General Meeting, 18-22 June 2006, Montreal, Canada.

[15] Nicolet, C., Arpe, J., and Avellan, F., 2004. "Identification and modeling of pressure fluctuations of a francis turbine scale model at part load operation". In Proceedings of the 22nd IAHR Symposium on Hydraulic Machinery and Systems, Stockholm, Sweden,.

[16] Wylie, E., and Streeter, V., 1983. Fluid transients. Feb Press, Michigan.

[17] Nicolet, C., 2007. "Hydroacoustic modelling and numerical simulation of the unsteady operation of hydroelectric systems". PhD thesis, EPFL. No. 3751.

[18] Foldyna, J., Sitek, L., and Haban, V., 2006. "Acoustic wave propagation in high-pressure system". Ultrasonics, 44(Supplement 1), December, pp. e1457-e1460.

[19] Wang, D., and Johnson, C., 1989. "Comparison study of some impulse-response identification methods". In Proceedings of the Annual Southeastern Symposium on System Theory, pp. 52-56. 